\title{
The Predictive Value of Epidermal Growth Factor (EGF) in Chronic and Acute Forms of Glomerulonephritis
}

ISSN: 2578-0395

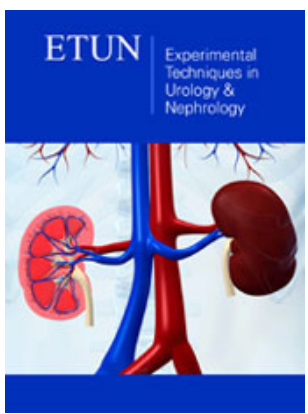

For HTML Version scan this QR code:

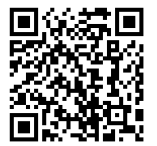

${ }^{* 1}$ Corresponding author: Maria Stangou, Department of Nephrology, Greece

Submission: 漈January 31, 2019

Published: ㄴ-1) March 05, 2019

Volume 2 - Issue 4

How to cite this article: Stangou $\mathrm{M}$ Sampani E, Daikidou D, Arseniou P, Stai $S$, et al. The Predictive Value of Epidermal Growth Factor (EGF) in Chronic and Acute Forms of Glomerulonephritis. Exp Tech Urol Nephrol. 3(4).ETUN.000546.2019. DOI: 10.31031/ETUN.2019.02.000546

Copyright@ Maria Stangou, This article is distributed under the terms of the Creative Commons Attribution 4.0 International License, which permits unrestricted use and redistribution provided that the original author and source are credited.

\author{
Stangou $\mathrm{M}^{1 *}$, Sampani E ${ }^{1}$, Daikidou $\mathrm{D}^{1}$, Arseniou $\mathrm{P}^{3}$, Stai $\mathrm{S}^{3}$, Nikolaidou $\mathrm{C}^{4}$, \\ Stamos $\mathrm{C}^{4}$ and Papagianni $\mathrm{A}^{1}$ \\ ${ }^{1}$ Department of Nephrology, Greece \\ ${ }^{2}$ Department of Immunology, Greece \\ ${ }^{3}$ Medical School, Greece \\ ${ }^{4}$ Department of Pathology, Greece
}

\section{Abstract}

Introduction and aim: EGF acts through EGF receptor and facilitates regeneration of tubular epithelial cells after injury. We investigated the role of urinary EGF excretion as biomarker for pathology and renal function outcome in glomerulonephritis.

Methods: EGF urinary levels were estimated in 3 forms of glomerular diseases:

A. IgA nephropathy (IgAN), as chronic glomerulonephritis, [n=50, 21 female, age 39.8yrs (1865)]

B. Pauci immune rapidly progressive glomerulonephritis (RPGN), as acute glomerulonephritis, [n=38, 17 female, age 59.5yrs (25-80)]

C. Nephrotic syndrome (NS) due to focal segmental glomerulosclerosis (FSGS) [ $\mathrm{n}=23,9$ female, age 47.5yrs(19-79)] and minimal change disease (MCD) [ $n=12$, 7female, age 45.5yrs (37-62)]. Ten healthy volunteers were used as controls. First morning urine samples were collected on the day of renal biopsy. Patients were followed up for 7.5 $\pm 2.1 \mathrm{yrs}$.

Result: EGF urinary levels were: IgAN $0.13 \pm 0.2$, FSGS $0.19 \pm 0.2$, MCD $0.7 \pm 0.4$, RPGN $0.15 \pm 0.3$, controls $0.14 \pm 0.07 \mathrm{pg} / \mathrm{mgUcr}$. EGF urinary levels had significant negative correlation with severity of interstitial fibrosis, $r=-0.6, p=0.02$, in IgAN, with the percentage of fibrous crescents, $r=-0.6, p=0.01$ in RPGN, and finally, with the percentage of global sclerosed glomeruli, $r=-0,5, p=0,04$, degree of fibrosis, $r=-$ $0.6, p=0.005$ and interstitial infiltration, $r=-0.6, p=0.01$ in patients with NS. Declining renal function was associated with reduced urinary EGF levels in IgAN $(0.04 \pm 0.04$ vs. $0.2 \pm 0.2 \mathrm{pg} / \mathrm{mgUcr}, \mathrm{p}=0.01)$, and FSGS ( $0.007 \pm 0.004$ vs. $0.6 \pm 0.04 \mathrm{pg} / \mathrm{mgUcr}, \mathrm{p}=0.009)$, but not in RPGN $(0.05 \pm 0.1$ vs. $0.2 \pm 0.4 \mathrm{pg} / \mathrm{mg} \mathrm{cr}, \mathrm{p}=\mathrm{NS})$.

Conclusion: Reduction of EGF urinary levels in patients with glomerulonephritis is associated with "chronic" histological changes, and can predict renal function outcome mainly in patients with chronic forms of glomerulonephritis.

\section{Introduction}

Glomerular diseases usually present with proteinuria, microscopic hematuria and renal function impairment. The above triad of clinical symptoms cannot discriminate between the wide range of different histological types of glomerular and/or tubular disorders. Renal biopsy remains the "gold standard" procedure for the diagnosis and validation of glomerular diseases, however, it is an invasive method, potentially followed by side effects, and cannot be routinely performed during patients follow up. Discovery of urinary biomarkers, capable to differentiate a disease, reflect severity of renal damage and predict outcome of renal function, has been the main scope for many years of research [1,2]. One such biomarker is the EGF, a peptide growth factor, which is produced by tubular epithelial cells and is excreted in the urine [3]. 
EGF acts as a tubular cell survival factor and plays an imperative role in regenerating tubular epithelial cells and restoring barrier function in the healing phase of renal injury. Thus, it protects epithelial cells during kidney injury and indeed its expression within the kidney is decreased in several kidney diseases [4-7]. Aim of the present study was to assess the urinary excretion of EGF in different forms of chronic and acute glomerular diseases, to correlate these levels with the severity of histologic findings and estimate the predictive role in the course of different forms of glomerulonephritis.

\section{Patients-Methods}

One hundred twenty-three patients were prospectively studied. All patients were diagnosed with primary glomerular diseases, IgAN ( $n=50)$, Focal Segmental Necrotizing Glomerulonephritis (FSGN, n=38), Focal Segmental Glomerulosclerosis (FSGS, n=23) and Minimal Change Nephropathy (MCN, $n=12)$. Diagnosis in all patients was based on renal biopsy findings. Patients with systemic immune diseases, such as Systemic Lupus Erythematosus or Rheumatoid Arthritis, with infections or neoplasia's, were excluded from the study.

First morning urine samples were collected the day of renal biopsy under sterile conditions, centrifuged at $252 \mathrm{~g}$ for $10 \mathrm{~min}$ and keptat $-70^{\circ} \mathrm{C}$, until used. A sandwich enzyme-linked immunosorbent assay (ELISA) method was applied for the measurement of EGF. Thirty healthy individuals were used as controls. Urine cytokine levels were normalized for urine creatinine (UCR) concentration and were expressed as pg/mg Ucr. Renal biopsies were evaluated for the severity of tubular atrophy, interstitial fibrosis, and frequency of crescent formation and global sclerosed glomeruli.

\section{Result}

Clinical characteristics from patients are shown in Table 1 and levels of urinary EGF in different forms of glomerulopathies are depicted in Figure 1.

Table 1: Clinical characteristics of patients at time of diagnosis.

\begin{tabular}{|c|c|c|c|c|}
\hline & $\operatorname{IgAN} n=50$ & $\mathrm{FSNG} n=38$ & FSGS $n=23$ & MCN $n=12$ \\
\hline Age(yrs) & $45.5 \pm 18$ & $54.5 \pm 15$ & $47.5 \pm 16.9$ & $45.5 \pm 9.3$ \\
\hline $\mathrm{M} / \mathrm{F}$ & $35 / 15$ & $16 / 17$ & $15 / 8$ & $07 / 5$ \\
\hline Screat (mg/dl) & $1.6 \pm 0.8$ & $3.8 \pm 2.5$ & $2.1 \pm 0.9$ & $0.8 \pm 0.2$ \\
\hline GFR $\left(\mathrm{ml} / \min 1.73 \mathrm{~m}^{2}\right)$ & $69 \pm 36.4$ & $23.6 \pm 18$ & $52.4 \pm 31.2$ & $107.2 \pm 31.6$ \\
\hline Upr (g/24hr) & $1.3 \pm 1.2$ & $1.4 \pm 0.9$ & $3.2 \pm 1.9$ & $4.9 \pm 3.3$ \\
\hline Urinary EGF (pg/mg Ucr) & $0.13 \pm 0.2$ & $0.15 \pm 0.3$ & $0.2 \pm 0.2$ & $0.7 \pm 0.4$ \\
\hline
\end{tabular}

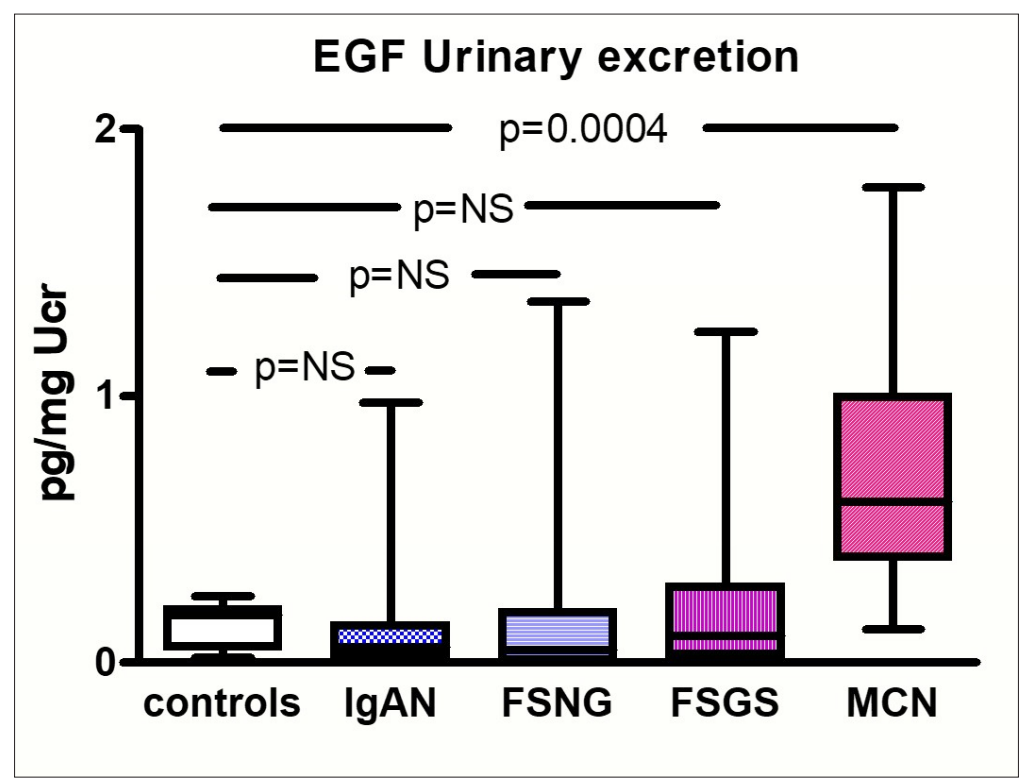

Figure 1: EGF urinary excretion in different forms of glomerular diseases, differences with healthy controls.

Correlations of EGF Urinary Levels with Laboratory and Histological Findings at Time of Renal Biopsy-Diagnosis

In patients with IgAN and FSGS/MCN urinary excretion of EGF had negative correlation with the severity of TIN fibrosis, $\mathrm{p}=0.02$ and $\mathrm{p}=0.005$, respectively. In patients with FSNG there was no correlation between EGF urinary levels and degree of tubulointerstitial levels, but there was significant negative correlation with the percentage of global sclerosed glomeruli and fibrous crescents, $p=0.01$ and $p=0.001$, respectively, (Table 2) and (Figure 2). 
Table 2: Correlations of EGF urinary levels with severity of histologic lesions.

\begin{tabular}{|c|c|c|c|c|c|c|}
\hline \multirow[t]{2}{*}{ Correlation of EGF with Histology } & \multicolumn{2}{|c|}{ IgAN } & \multicolumn{2}{|c|}{ FSNG } & \multicolumn{2}{|c|}{ FSGS/MCN } \\
\hline & $\mathrm{r}$ & $\mathrm{p}$ & $\mathrm{r}$ & $\mathrm{p}$ & $\mathrm{r}$ & $\mathrm{p}$ \\
\hline Global sclerosis (\%) & & NS & -0.5 & 0.01 & -0.5 & 0.04 \\
\hline Fibrous crescents (\%) & & NS & -0.6 & 0 & & \\
\hline Int. fibrosis (\%) & -0.6 & 0.02 & & NS & -0.6 & 0.005 \\
\hline Int. infiltration & & NS & & NS & -0.6 & 0.01 \\
\hline
\end{tabular}
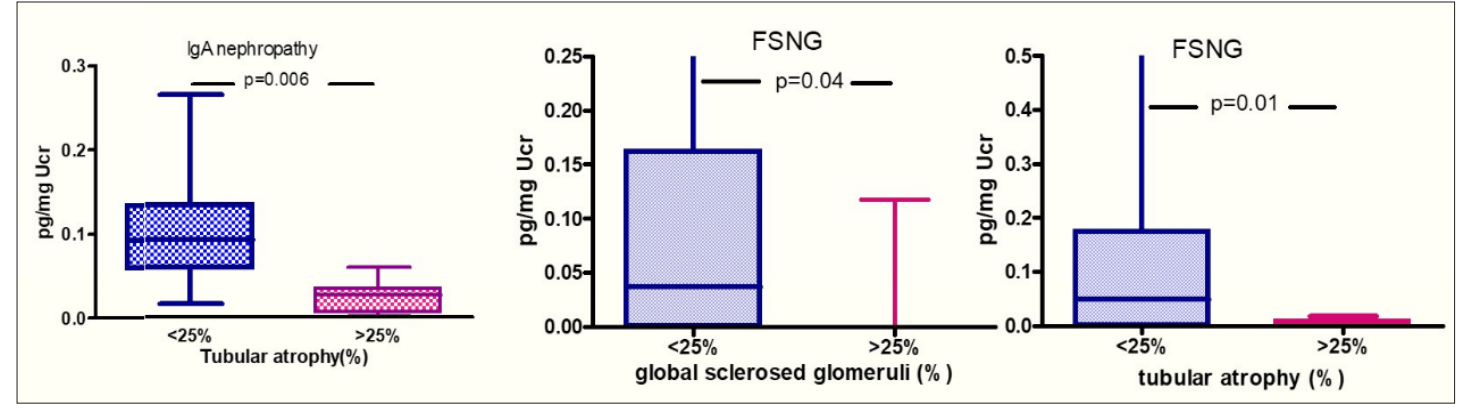

Figure 2: Differences in EGF urinary levels, according to different histologic findings.

EGF Urinary Excretion as a Predictive Factor to Renal Function Outcome

EGF urinary levels had significant correlations with eGFR at last follow up. FSGS patients were separated to progressors and non-progressors, according to their renal function outcome, EGF urinary levels at time of renal biopsy were significantly lower to progressors compared to those who retained renal function (Figure $3 \& 4)$.

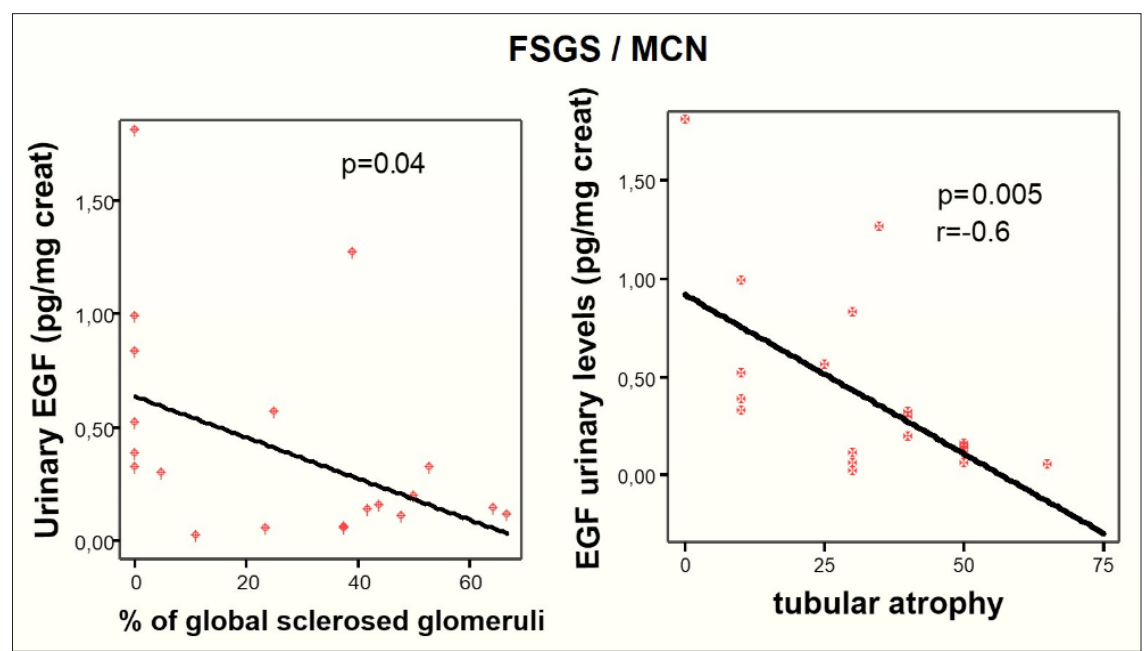

Figure 3: Correlation of EGF urinary levels with percentage of global sclerosed glomeruli and degree of tubular atrophy in patients with nephrotic syndrome (due to FSGS and MCN).
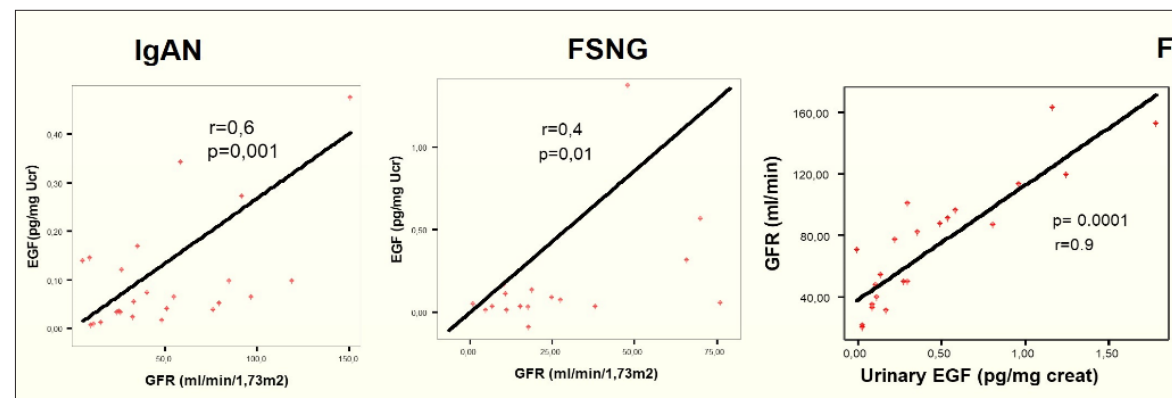

FSGS

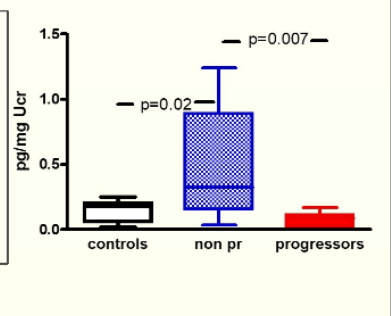

Figure 4: Correlation of EGF urinary levels with renal function outcome. 


\section{Discussion}

Primary glomerular diseases have different etiologies, immune reactions, histology, and different outcome of renal function. Primary glomerular diseases are characterized by the presence of acute and/or chronic lesions. Common acute lesions include endothelial hyperplasia, focal necrosis, cellular crescent formation, inflammatory infiltration, while chronic lesions consist of focal or global sclerosis, fibrous crescents and tubulointerstitial fibrosis.

IgAN is characterized by the presence of both acute and chronic lesions, however the vast majority of patients develop chronic renal pathology and progress slowly to renal function impairment. FSNG is the typical form of acute glomerulonephritis, characterized by necrosis and extra capillary proliferation. MCN and FSGS are the principal causes of nephrotic syndrome, and they also share many histological findings. Podocyte injury and fusion of foot processes, with or without podocyte hypertrophy and hyperplasia, and only scarce inflammatory findings are common findings in both diseases and so, differential diagnosis between FSGS and MCN is usually difficult [7]. Description of biomarkers definitely associated with particular histologic findings should be extremely useful in distinguishing between FSGS and MCN and predicting renal function outcome in other types of glomerulonephritis [8].

In the present study there was significant reduction in urinary levels of EGF in FSGS patients, compared to MCN, which can discriminate the two entities. More interestingly, there was also a negative correlation with the degree of glomerulosclerosis, fibrous crescent formation and TIN fibrosis. Furthermore, urinary levels of EGF at time of diagnosis could predict long term renal function outcome of all types of glomerulonephritis included in the study.

EGF is produced by epithelial cells, it stimulates the activation of nuclear factor (NF)-kB and cyclin D1 expression in human proximal tubular cells and thus, participates in the regulation of cell proliferation [9].

EGF urinary levels at time of diagnosis, in all four types of glomerular diseases studied, showed a negative correlation with chronic histologic findings in renal biopsy and also, they seemed to have a predictive value in renal function outcome, as EGF excretion was significantly lower in patients who progressed during follow up compared with those patients whose renal function remained stable. It could be a simplified explanation, advocating that given the tubular origin of EGF, its production will be eliminated in cases of tubular atrophy, seen in advanced stages of renal impairment. However, EGF receptor which is expressed at tubular epithelial cells, after activation by urinary proteins, seems to have a central role in this procedure. EGFR is used by EGF and TGF- $\beta$, which antagonize for the receptor, and also, have opposite results [10]. Our results suggest that EGF urinary levels are able to discriminate between MCN and FSGS, and also predict renal function outcome in acute and chronic forms of primary glomerulopathies.

\section{References}

1. D'Agati VD, Mengel M (2013) The rise of renal pathology in nephrology: Structure illuminates' function. Am J Kidney Dis 61(6): 1016-1025.

2. Konvalinka A, Scholey JW, Diamandis EP (2012) Searching for new biomarkers of renal diseases through proteomics. Clin Chem 58(2): 353365 .

3. Worawichawong S, Radinahamed P, Muntham D, Sathirapongsasuti N, Nongnuch A, et al. (2016) Urine epidermal growth factor, monocyte chemoattractant protein-1 or their ratio as biomarkers for interstitial fibrosis and tubular atrophy in primary glomerulonephritis. Kidney Blood Press Res 41(6): 997-1007.

4. Lechner J, Malloth NA, Jennings P, Heckl D, Pfaller W, et al. (2007) Opposing roles of EGF in IFN-alpha-induced epithelial barrier destabilization and tissue repair. Am J Physiol Cell Physiol 293(6): C1843-C1850.

5. Satirapoj B, Dispan R, Radinahamed P, Kitiyakara C (2018) Urinary epidermal growth factor, monocyte chemoattractant protein-1 or their ratio as predictors for rapid loss of renal function in type 2 diabetic patients with diabetic kidney disease. BMC Nephrol 19(1): 246.

6. Gesualdo L, Paolo DS, Calabro A, Milani S, Maiorano E, et al. (1996) Expression of epidermal growth factor and its receptor in normal and diseased human kidney: an immunohistochemical and in situ hybridization study. Kidney Int 49(3): 656-665.

7. Maas RJ, Deegens JK, Smeets B, Moeller MJ, Wetzels JF (2016) Minimal change disease and idiopathic FSGS: manifestations of the same disease. Nat Rev Nephrol 12(12): 768-776.

8. Königshausen E, Sellin L (2016) Circulating permeability factors in primary focal segmental glomerulosclerosis: A review of proposed candidates. Biomed Res Int 2016: 3765608.

9. Chen ZD, Xu L, Tang KK, Gong FX, Liu JQ, et al. (2016) NF-kB-dependent transcriptional upregulation of cyclin D1 exerts cytoprotection against hypoxic injury upon EGFR activation. Exp Cell Res 347(1): 52-59.

10. Samarakoon R, Dobberfuhl AD, Cooley C, Overstreet JM, Patel S, et al. (2013) Induction of renal fibrotic genes by TGF- $\beta 1$ requires EGFR activation, p53 and reactive oxygen species. Cell Signal 25(11): 2198-2209.

For possible submissions Click below: 